\title{
FONTES DE INFORMAÇÃO E AVALIAÇÃO DE CONHECIMENTOS SOBRE ALIMENTA- ÇÃO E NUTRIÇÃO EM COLABORADORES DE UM MUNICÍPIO
}

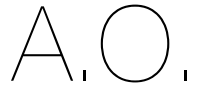

ARTIGO ORIGINAL

\section{Faculdade de Ciências da} Nutrição e Alimentação da Universidade do Porto, Rua do Campo Alegre, n. ${ }^{\circ} 823$,

4150-180 Porto, Portugal

${ }^{2}$ Câmara Municipal da Maia, Pelouro da Saúde Gabinete de Saúde,

Praça do Doutor José Vieira de Carvalho, 4474-006 Maia, Portugal

${ }^{3}$ EPIUnit - Instituto de Saúde Pública da Universidade do Porto, Rua das Taipas, n. ${ }^{\circ} 135$ 4050-600 Porto, Portugal

\section{*Endereço para}

Cláudia Pessoa

Faculdade de Ciências da Nutrição e Alimentação da Universidade do Porto, Rua do Campo Alegre, n. 823 4150-180 Porto, Portugal up201505706@fcna.up.pt

Histórico do artigo: Recebido a 22 de setembro

Recebid
de 2020

Aceite a 19 de novembro de 2020

\author{
SOURCES OF INFORMATION AND ASSESSMENT OF \\ NUTRITIONAL KNOWLEDGE AMONG EMPLOYEES OF A \\ CITY HALL
}

Cláudia Pessoa"; ${ }^{1 *}$ Marta Sampaio²; Rui Poínhos'; Cláudia Afonso

\section{RESUMO}

INTRODUÇÃo: O local de trabalho é uma importante área de ação para a promoção de estilos de vida saudáveis.

OBJETIVOS: Avaliar fontes de informação e temáticas que suscitam maior interesse sobre alimentação/nutrição, estado de saúde e conhecimentos sobre alimentação dos colaboradores de uma câmara municipal.

METODOLOGIA: Participaram no estudo 118 colaboradores de uma câmara municipal. Foram recolhidos dados sociodemográficos, autoperceção do estado de saúde, peso e altura, fontes de informação e temas preferenciais sobre alimentação/nutrição e conhecimentos nesta área (recomendações dietéticas; fonte alimentar de nutrientes; relação dieta-doença) através do Questionário de Conhecimentos Nutricionais (QCN).

RESULTADOS: As mulheres apresentaram nível superior de conhecimentos, quer no total ( $p=0,001)$, quer nas secções relativa à fonte alimentar de nutrientes $(p=0,001)$ e à relação dieta-doença $(p=0,030)$. Também os participantes mais novos $(R=-0,205$; $p=0,030)$, com menor índice de massa corporal $(R=-0,188 ; p=0,049)$, maiores habilitações literárias $(R=0,506 ; p<0,001)$ e que se autopercecionam mais saudáveis $(R=0,123)$ apresentaram nível superior de conhecimentos. A secção relativa à relação dietadoença foi aquela em que se verificou menor nível de conhecimentos. Relativamente às fontes de informação, $67,8 \%$ relataram ter por hábito fazê-lo, principalmente na Internet (58,5\%) ou através de um médico (30,5\%). Os participantes que realizam pesquisa apresentaram um maior nível de conhecimentos (mediana =67,2\% vs. 57,8\%; $p=0,004$ ). Estudaram-se também as diferenças nos conhecimentos entre quem utilizava ou não cada fonte de informação.

CONCLUSõES: Estes resultados evidenciam que o nível de conhecimentos sobre alimentação neste grupo não é elevado, justificando a necessidade de implementar intervenções a nível da promoção da literacia alimentar/nutricional. Pelas suas características, a vertente da educação alimentar deve ser prioritária, bem como intervenções ao nível do ambiente alimentar no local de trabalho.

\section{PALAVRAS-CHAVE}

Conhecimentos alimentares e nutricionais, Intervenções alimentares, Local de trabalho, Promoção da saúde

ABSTRACT

INTRODUCTION: The workplace is an important area of action for the promotion of healthy lifestyles.

OBJECTIVES: To evaluate sources of information and food/nutrition topics which arouse greatest interest, health status and nutritional knowledge among employees of a city hall.

METHODOLOGY: One hundred eighteen employees of a city hall participated in the study. Sociodemographic data, self-perception of health status, weight and height, sources of information and preferential food/nutrition issues, and knowledge on this subject (dietary recommendations; food source of nutrients; diet-disease relationship) were obtained through the Nutritional Knowledge Questionnaire (NKQ).

RESULTS: Females had higher level of knowledge, either in total $(p=0.001)$ and in the sections on food source of nutrients $(p=0.001)$ and on diet-disease relationship $(p=0.030)$. Also, younger participants $(R=-0.205 ; p=0.030)$, those with lower body mass index $(R=-0.188 ; p=0.049)$, those with higher academic qualifications $(R=0.506 ; p<0.001)$ and those who perceived themselves as healthy $(R=0.123)$ had higher level of knowledge. The section on diet-disease relationship registered the lowest level of knowledge. Regarding sources of information, $67.8 \%$ reported the habit of doing it, mainly using the Internet (58.5\%) and through a doctor (30.5\%). Employees who research information had higher level of knowledge (median $=67.2 \%$ vs. 57.8\%; $p=0.004$ ). We also studied the differences on knowledge between participants who use or not each information source.

CONCLUSIONS: These results show that, in this group, the level of knowledge about food/nutrition is not high, justifying the need to implement interventions in terms of promoting food/nutritional literacy. Given its characteristics, food education should be a priority, as well as interventions focusing food environment on the workplace.

KEYWORDS

Nutritional knowledge, Dietary interventions, Workplace, Health promotion 


\section{INTRODUÇÃO}

As doenças crónicas não transmissíveis (DCNT) são a principal causa de morte a nível europeu e, em particular, em Portugal (1, 2). $\mathrm{Na}$ origem destas doenças encontram-se diversos fatores de risco modificáveis, dos quais se destacam os hábitos alimentares inadequados, a hipertensão arterial e o Índice de Massa Corporal (IMC) elevado (1, 3-5). Torna-se, por isso, fundamental a promoção de hábitos alimentares saudáveis para a prevenção do desenvolvimento de doenças e promoção da qualidade de vida (6).

As condições de vida e de trabalho, concretamente o ambiente de trabalho, são consideradas básicas para a saúde do indivíduo. Assim, o ambiente de trabalho torna-se num local de eleição para a promoção de estilos de vida saudáveis $(2,7)$. Neste sentido, a avaliação de conhecimentos e práticas alimentares deverá ser uma preocupação por parte dos responsáveis pelos locais de trabalho. Resultados da investigação demonstram, de forma clara, que colaboradores com obesidade apresentam uma maior prevalência de absentismo que os demais $(8,9)$.

Em Portugal, aproximadamente $78 \%$ da população economicamente ativa, com mais de 15 anos, trabalha, sendo que a maior parte se encontra com um contrato de trabalho a tempo integral (10), o que significa que passa grande parte do seu dia no posto de trabalho. Abre-se, assim, uma janela de oportunidades para o desenvolvimento e implementação de ações de saúde pública num local privilegiado, tendo como desafio a grande diversidade a nível socioeconómico, de estado geral de saúde e, também, cultural (9).

O local de trabalho é já reconhecido pela Organização Mundial da Saúde (OMS) como uma importante área de ação para a promoção de comportamentos alimentares saudáveis, contribuindo para a prevenção de DCNT na população ativa, surgindo a possibilidade de os transpor para a vida familiar $(9,11)$. Neste local, existem diversas estratégias possiveis para influenciar os comportamentos de saúde, nomeadamente a nível da alimentação. Por um lado, capacitando os colaboradores com conhecimentos sobre como praticar uma alimentação saudável e, por outro, modificando os ambientes alimentares e potenciando a mobilização desses conhecimentos $(12,13)$.

\section{OBJETIVOS}

Avaliar as fontes de informação e temáticas que suscitam maior interesse sobre alimentação/nutrição, a perceção do estado de saúde e os conhecimentos sobre alimentação dos colaboradores de uma autarquia.

\section{METODOLOGIA}

Neste estudo foi utilizada uma amostra de conveniência, sendo convidados a participar 306 colaboradores de uma câmara municipal, tendo participado 126 (taxa de resposta de 41,2\%). Após exclusão de oito questionários por preenchimento incompleto, foram considerados para análise os dados de 118 colaboradores. A recolha de dados foi realizada em julho de 2019.

Foi construído um questionário de aplicação direta que englobava questões sociodemográficas (sexo, idade, habilitações literárias) e sobre o estado de saúde (autoperceção do estado de saúde, peso, altura). Incluíram-se, também, questões relativas à procura de informação sobre alimentação/nutrição e o interesse por diferentes áreas da alimentação/nutrição.

Para avaliar os conhecimentos sobre alimentação e nutrição, recorreu-se à versão curta de um questionário adaptado e validado para estudantes portugueses do ensino superior (14). Nesta versão, igualmente validada, exclui-se a Secção III da versão longa pela falta de consistência interna e de validade dos itens que poderia pôr em causa a validade e a consistência interna global da versão reduzida. As questões relativas à avaliação dos conhecimentos sobre alimentação estão divididas em 3 secções, tendo sido atribuída uma pontuação a cada secção: a secção I é relativa a recomendações dietéticas; a secção II refere-se à fonte alimentar de nutrientes e a secção IV aborda a relação dieta-doença. No final, por decisão dos autores, foi calculada a pontuação conjunta das 3 secções. Para a comparação do nível de conhecimentos entre os aspetos avaliados pelas diferentes secções, os resultados foram convertidos em percentagem de respostas corretas. Previamente à recolha de informação, foi realizado um teste piloto numa amostra de cinco pessoas de diferentes níveis socioeconómicos e académicos. Este teste permitiu testar as perguntas e a compreensão do questionário, resultando em reformulações. O protocolo obteve parecer favorável da Comissão de Ética da Universidade do Porto e neste trabalho foram considerados os princípios presentes na Declaração de Helsínquia, tendo sido garantida a proteção e confidencialidade de todas as informações recolhidas.

O tratamento estatístico foi realizado no programa IBM ${ }^{\circledR}$ SPSS ${ }^{\circledR}$ versão 25.0 para Windows ${ }^{\circledR}$. A estatística descritiva consistiu no cálculo de frequências absolutas (n) e relativas (\%), de medianas e de percentis (P25; P75). A normalidade das variáveis cardinais foi estudada pelos coeficientes de simetria e de achatamento. O grau de associação entre pares de variáveis foi medido pelo coeficiente de correlação de Spearman (R). O teste de Mann-Whitney foi utilizado para comparar ordens médias de pares de amostras independentes e o teste de Friedman para comparar ordens médias de 3 amostras emparelhadas; quando o teste de Friedman indicou diferenças significativas, foram realizados testes post-hoc (Wilcoxon), com correção de Bonferroni. O teste t-Student foi utilizado para comparar as médias de pares de amostras independentes. Rejeitou-se a hipótese nula quando $p<0,05$.

\section{RESULTADOS}

A amostra deste estudo é constituída por 118 colaboradores da autarquia, sendo a maioria do sexo feminino (55,9\%). A mediana das idades dos que indicaram esta informação $(n=112)$ é de 48 anos (P25 = 42; P75 = 54)

No que diz respeito à autoperceção do seu estado de saúde, nove participantes consideram-se pouco saudáveis $(7,6 \%), 18$ consideram-se nem saudáveis nem doentes (15,3\%), 79 relatam ser saudáveis (66,9\%) e 12 participantes consideram-se muito saudáveis (10,2\%). Há diferenças entre o nível de conhecimentos das 3 secções $(p<0,001)$. Os testes post-hoc mostraram menor nível de conhecimentos sobre a relação dieta-doença (mediana $=44,4 \%$; P25 = 27,8; P75 = 61,1) do que sobre recomendações dietéticas (mediana $=83,3 \%$; P25 = 66,7; P75 = 83,3; $p<0,001$ ) e do que sobre fonte alimentar de nutrientes ( mediana $=73,5 \% ;$ P25 = 55,9; P75 = 82,4; p < 0,001).

Na Tabela 1 compara-se o nível de conhecimentos (total e nas diferentes secções) entre mulheres e homens. As mulheres apresentaram maior nível de conhecimentos, quer em termos de pontuação total ( $p=0,001)$, quer nas secções relativa à fonte alimentar de nutrientes $(p=0,001)$ e relativa à relação dieta-doença $(p=0,030)$.

Para além disso, o nível na pontuação total foi tanto maior quanto menor a idade $(R=-0,205 ; p=0,030)$ e o IMC $(R=-0,188 ; p=0,049)$ e quanto maiores as habilitações literárias $(R=0,506 ; p<0,001)$ e a autoperceção do estado de saúde $(R=0,123)$.

$\mathrm{Na}$ Tabela 2, estão descritas as frequências das temáticas que suscitam maior interesse na área da alimentação/nutrição, sendo que se destacam a culinária saudável (72,9\%), a alimentação mediterrânica $(30,5 \%)$ e a leitura de rótulos alimentares $(24,6 \%)$. 
No que diz respeito à pesquisa por informação sobre alimentação/ nutrição, 80 participantes $(67,8 \%)$ relataram ter o hábito de o fazer. Na Tabela 3, estão descritas as frequências das fontes de informação onde realizam essa pesquisa.

Os participantes que realizam pesquisa sobre alimentação/nutrição apresentaram um maior nível de conhecimentos comparativamente com quem não pesquisa (mediana $=67,2 \%$ vs. 57,8\%; $p=0,004$ ), apresentando também diferenças estatisticamente significativas para a secção relativa a fontes alimentares de nutrientes (mediana $=76,5 \%$ vs. $61,8 \% ; p=0,006$ ) e para a secção sobre a relação dieta-doença (mediana $=50,0 \%$ vs. 36,1\%; $p=0,017$ ).

$\mathrm{Na}$ Tabela 4, compara-se o nível de conhecimentos (total e nas diferentes secções) entre quem refere ou não utilizar cada uma das fontes de informação sobre alimentação/nutrição mais frequentes.

Relativamente à fonte de informação mais usada, a Internet, os participantes que a usam não diferem significativamente dos restantes em termos de conhecimentos. Os participantes que referiram obter informação nesta área através de um médico apresentaram diferenças estatisticamente significativas na secção que aborda a relação dietadoença $(p=0,032)$. Para além disso, os participantes que referiram obter informação através de um nutricionista apresentaram diferenças estatisticamente significativas em relação às recomendações dietéticas $(p=0,035)$.

\section{DISCUSSÃO DOS RESULTADOS}

No presente estudo, os indivíduos do sexo feminino, os que têm menor idade e os que têm um IMC mais baixo apresentaram, no geral, um nível de conhecimentos superior, relativamente à alimentação. A literatura evidencia também a associação negativa entre um maior nível de conhecimentos e obesidade, considerando que, eventualmente, os conhecimentos sobre alimentação saudável poderão conduzir a melhores escolhas alimentares e, consequentemente, a uma redução na prevalência de obesidade $(15,16)$.

Relativamente à pesquisa por informação sobre alimentação/nutrição, a grande maioria usa a Internet, tal como verificado em outros estudos $(17,18)$, seguido da consulta com o médico e familiares, colegas e/ou amigos. De facto, a Internet é uma plataforma importante para a partilha de informação sobre saúde e nutrição por instituições de saúde pública. Contudo, também é possível encontrar-se diversos sítios eletrónicos e fóruns sobre saúde e nutrição cujo conteúdo nem sempre é fidedigno ou certificado por especialistas (19-21). Apesar de a principal fonte de informação ser a Internet, foi possível observar que os sítios eletrónicos de Organizações Governamentais e sítios eletrónicos de Associações Profissionais e Sociedades Científicas apresentam pouca adesão, com cerca de $7 \%$ cada. Estes dados podem indicar que a maioria da pesquisa realizada pelos colaboradores na Internet é feita em sítios eletrónicos, fóruns ou redes sociais, onde a qualidade e a confiabilidade da informação em saúde prestada variam substancialmente, tal como é possível observar em estudos internacionais (22-24) e nacionais (25). Todavia, um estudo sobre este tema relatou que a Internet apenas serve como complemento de informação nestas áreas e não como substituto de outras fontes, como profissionais de saúde, familiares ou colegas (26). Outro estudo revelou que dois em cada cinco portugueses afirmam que essa informação os levou a fazer sugestões ou a colocar questões ao profissional de saúde (27).

No que diz respeito à relação entre o nível de conhecimentos e a pesquisa na Internet, verificaram-se níveis superiores de conhecimentos, o que vai ao encontro do que foi observado num estudo realizado também em Portugal (28), podendo ser explicada pelo facto de a Internet permitir um acesso fácil e a baixo custo à informação. No entanto, a

\section{Tabela 1}

Relação entre o nível de conhecimentos e o sexo

\begin{tabular}{|c|c|c|c|c|c|c|c|c|}
\hline & \multicolumn{2}{|c|}{$\begin{array}{l}\text { RECOMENDAÇõES DIETÉTICAS } \\
(\%)\end{array}$} & \multicolumn{2}{|c|}{$\begin{array}{l}\text { FONTE ALIMENTAR DE NUTRIENTES } \\
(\%) \\
\end{array}$} & \multicolumn{2}{|c|}{$\begin{array}{l}\text { RELAÇÃO DIETA-DOENÇA } \\
(\%)\end{array}$} & \multicolumn{2}{|c|}{$\begin{array}{c}\text { TOTAL } \\
(\%)\end{array}$} \\
\hline & $\begin{array}{l}\text { MEDIANA } \\
\text { (P25; P75) }\end{array}$ & p & $\begin{array}{l}\text { MEDIANA } \\
\text { (P25; P75) }\end{array}$ & $p$ & $\begin{array}{l}\text { MEDIANA } \\
\text { (P25; P75) }\end{array}$ & $\mathrm{p}$ & $\begin{array}{l}\text { MEDIANA } \\
\text { (P25; P75) }\end{array}$ & p \\
\hline Mulheres & $\begin{array}{c}83,3 \\
(66,7: 83,3)\end{array}$ & \multirow{2}{*}{0,415} & $\begin{array}{c}76,5 \\
(64,7 ; 85,3)\end{array}$ & \multirow{2}{*}{0,001} & $\begin{array}{c}47,2 \\
(33,3 ; 68,1)\end{array}$ & \multirow{2}{*}{0,030} & $\begin{array}{c}68,1 \\
(58,6 ; 75,9)\end{array}$ & \multirow{2}{*}{0,001} \\
\hline Homens & $\begin{array}{c}83,3 \\
(66,7 ; 83,3)\end{array}$ & & $\begin{array}{c}60,3 \\
(39,0 ; 81,6)\end{array}$ & & $\begin{array}{c}38,9 \\
(22,2 ; 55,6)\end{array}$ & & $\begin{array}{c}58,6 \\
(37,5 ; 69,0)\end{array}$ & \\
\hline
\end{tabular}

Tabela 2

Temáticas que suscitam maior interesse sobre nutrição/alimentação

\begin{tabular}{lc} 
& $n(\%)$ \\
Culinária saudável & $86(72,9)$ \\
\hline Alimentação mediterrânica & $36(30,5)$ \\
\hline Leitura de rótulos alimentares & $29(24,6)$ \\
\hline Alimentação sustentável & $23(19,5)$ \\
\hline Nutrição nas diferentes fases do ciclo de vida & $21(17,8)$ \\
\hline Alimentação vegetariana & $19(16,1)$ \\
\hline Nutrição em caso de patologia & $17(14,4)$ \\
\hline Nutrição no desporto & $16(13,6)$ \\
\hline Alergias e intolerâncias alimentares & $15(12,7)$ \\
\hline "Super alimentos" & $10(8,5)$ \\
\hline Novos alimentos e padrões alimentares & $8(6,8)$ \\
\hline Dietas da "moda" & $5(4,2)$ \\
\hline Aditivos alimentares & $2(1,7)$ \\
\hline
\end{tabular}

* A soma das percentagens é superior a 100, pois os inquiridos podiam referir mais do que uma hipótese de escolha.
Tabela 3

Fontes de informação habituais sobre nutrição/alimentação

\begin{tabular}{ll} 
& $n(\%)$ \\
Internet & $69(58,5)$ \\
\hline Consulta com o médico & $36(30,5)$ \\
\hline Familiares, colegas e/ou amigos & $35(29,7)$ \\
\hline Jornais, revistas e/ou panfletos & $27(22,9)$ \\
\hline Consulta com o nutricionista ou dietista & $22(18,6)$ \\
\hline Livros & $22(18,6)$ \\
\hline Programas de televisão & $19(16,1)$ \\
\hline Publicações científicas & $16(13,6)$ \\
\hline Sítios eletrónicos de Organizações Governamentais & $8(6,8)$ \\
\hline Sítios eletrónicos de Associações Profissionais e & $8(6,8)$ \\
\hline Sociedades Científicas & $6(5,1)$ \\
\hline Cursos e palestras & $4(3,4)$ \\
\hline Consulta com outro profissional de saúde & $1(0,8)$ \\
\hline Programas de rádio &
\end{tabular}

* A soma das percentagens é superior a 100, pois os inquiridos podiam referir mais do que uma hipótese de escolha. 
Relação entre o nível de conhecimentos e as fontes de informação mais frequentes sobre nutrição/alimentação

\begin{tabular}{|c|c|c|c|c|c|c|c|c|c|}
\hline & & \multicolumn{2}{|c|}{$\begin{array}{l}\text { RECOMENDAÇÕES DIETÉTICAS } \\
(\%)\end{array}$} & \multicolumn{2}{|c|}{$\begin{array}{l}\text { FONTE ALIMENTAR DE NUTRIENTES } \\
(\%)\end{array}$} & \multicolumn{2}{|c|}{$\begin{array}{l}\text { RELAÇÃO DIETA-DOENÇA } \\
(\%)\end{array}$} & \multicolumn{2}{|c|}{$\begin{array}{c}\text { TOTAL } \\
(\%)\end{array}$} \\
\hline & & $\begin{array}{l}\text { MEDIANA } \\
\text { (P25; P75) }\end{array}$ & p & $\begin{array}{l}\text { MEDIANA } \\
\text { (P25; P75) }\end{array}$ & $p$ & $\begin{array}{l}\text { MEDIANA } \\
\text { (P25; P75) }\end{array}$ & p & $\begin{array}{l}\text { MEDIANA } \\
\text { (P25; P75) }\end{array}$ & p \\
\hline \multirow{2}{*}{ Internet } & Não & $\begin{array}{c}83,3 \\
(66,7 ; 83,3)\end{array}$ & \multirow{2}{*}{0,876} & $\begin{array}{c}70,6 \\
(56,6 ; 82,4)\end{array}$ & \multirow{2}{*}{0,394} & $\begin{array}{c}52,8 \\
(38,9 ; 70,8)\end{array}$ & \multirow{2}{*}{0,589} & $\begin{array}{c}66,4 \\
(48,3 ; 76,3)\end{array}$ & \multirow{2}{*}{0,739} \\
\hline & Sim & $\begin{array}{c}83,3 \\
(66,7 ; 83,3)\end{array}$ & & $\begin{array}{c}76,5 \\
(58,8 ; 85,3)\end{array}$ & & $\begin{array}{c}50,0 \\
(33,3 ; 61,1)\end{array}$ & & $\begin{array}{c}67,2 \\
(56,9 ; 74,1)\end{array}$ & \\
\hline \multirow{2}{*}{$\begin{array}{l}\text { Consulta com } \\
\text { o médico }\end{array}$} & Não & $\begin{array}{c}83,3 \\
(66,7 ; 83,3)\end{array}$ & \multirow{2}{*}{0,643} & $\begin{array}{c}76,5 \\
(55,9 ; 83,8)\end{array}$ & \multirow{2}{*}{0,170} & $\begin{array}{c}44,4 \\
(25,0 ; 61,1)\end{array}$ & \multirow{2}{*}{0,032} & $\begin{array}{c}65,5 \\
(46,6 ; 73,3)\end{array}$ & \multirow{2}{*}{0,056} \\
\hline & Sim & $\begin{array}{c}83,3 \\
(66,7 ; 83,3)\end{array}$ & & $\begin{array}{c}76,5 \\
(64,7 ; 85,3)\end{array}$ & & $\begin{array}{c}55,6 \\
(38,9 ; 70,8)\end{array}$ & & $\begin{array}{c}72,4 \\
(62,5 ; 75,4)\end{array}$ & \\
\hline \multirow{2}{*}{$\begin{array}{l}\text { Familiares, } \\
\text { colegas e/ou } \\
\text { amigos }\end{array}$} & Não & $\begin{array}{c}83,3 \\
(66,7 ; 83,3)\end{array}$ & \multirow{2}{*}{0,509} & $\begin{array}{c}76,5 \\
(58,1 ; 82,4)\end{array}$ & \multirow{2}{*}{0,242} & $\begin{array}{c}50,0 \\
(33,3 ; 66,7)\end{array}$ & \multirow{2}{*}{0,730} & $\begin{array}{c}65,5 \\
(53,4 ; 58,6)\end{array}$ & \multirow{2}{*}{0,354} \\
\hline & Sim & $\begin{array}{c}83,3 \\
(66,7 ; 83,3)\end{array}$ & & $\begin{array}{c}79,4 \\
(58,8 ; 88,2)\end{array}$ & & $\begin{array}{c}50,0 \\
(38,9 ; 61,1)\end{array}$ & & $\begin{array}{c}70,7 \\
(65,5 ; 75,9)\end{array}$ & \\
\hline \multirow{2}{*}{$\begin{array}{l}\text { Jornais, revistas } \\
\text { e/ou panfletos }\end{array}$} & Não & $\begin{array}{c}83,3 \\
(66,7 ; 83,3)\end{array}$ & \multirow{2}{*}{0,452} & $\begin{array}{c}76,5 \\
(58,8 ; 85,3)\end{array}$ & \multirow{2}{*}{0,972} & $\begin{array}{c}44,4 \\
(27,8 ; 61,1)\end{array}$ & \multirow{2}{*}{0,100} & $\begin{array}{c}66,4 \\
(51,3 ; 75,9)\end{array}$ & \multirow{2}{*}{0,627} \\
\hline & Sim & $\begin{array}{c}83,3 \\
(66,7 ; 83,3)\end{array}$ & & $\begin{array}{c}76,5 \\
(58,8 ; 85,3)\end{array}$ & & $\begin{array}{c}55,6 \\
(44,4 ; 66,7)\end{array}$ & & $\begin{array}{c}69,0 \\
(62,1 ; 74,1)\end{array}$ & \\
\hline \multirow{2}{*}{$\begin{array}{l}\text { Consulta com o } \\
\text { nutricionista ou } \\
\text { dietista }\end{array}$} & Não & $\begin{array}{c}83,3 \\
(66,7 ; 83,3)\end{array}$ & \multirow{2}{*}{0,035} & $\begin{array}{c}76,5 \\
(55,9 ; 82,4)\end{array}$ & \multirow{2}{*}{0,127} & $\begin{array}{c}50,0 \\
(33,3 ; 61,1)\end{array}$ & \multirow{2}{*}{0,319} & $\begin{array}{c}65,5 \\
(48,3 ; 74,1)\end{array}$ & \multirow{2}{*}{0,075} \\
\hline & Sim & $\begin{array}{c}83,3 \\
(88,3 ; 88,3)\end{array}$ & & $\begin{array}{c}80,9 \\
(64,7 ; 88,2)\end{array}$ & & $\begin{array}{c}55,6 \\
(38,9 ; 68,1)\end{array}$ & & $\begin{array}{c}71,6 \\
(63,8 ; 77,2)\end{array}$ & \\
\hline \multirow{2}{*}{ Livros } & Não & $\begin{array}{c}83,3 \\
(66,7 ; 83,3)\end{array}$ & \multirow{2}{*}{0,681} & $\begin{array}{c}76,5 \\
(58,8 ; 85,3)\end{array}$ & \multirow{2}{*}{0,440} & $\begin{array}{c}50,0 \\
(33,361,1)\end{array}$ & \multirow{2}{*}{0,252} & $\begin{array}{c}65,5 \\
(51,7 ; 74,1)\end{array}$ & \multirow{2}{*}{0,580} \\
\hline & Sim & $\begin{array}{c}83,3 \\
(62,5 ; 83,3)\end{array}$ & & $\begin{array}{c}77,9 \\
(62,2 ; 86,0)\end{array}$ & & $\begin{array}{c}52,8 \\
(38,9 ; 69,4)\end{array}$ & & $\begin{array}{c}67,2 \\
(59,9 ; 74,6)\end{array}$ & \\
\hline
\end{tabular}

discrepância entre níveis de conhecimentos não é significativa (Pesquisa na Internet: $\operatorname{Sim}=67,2 \%$; Não = 66,4\%) por ter origens muito distintas e, em função das características de quem a acede, poderá haver maior ou menor capacidade de seleção de informação fidedigna e veiculada por instituições e profissionais de saúde certificados (28). Sendo assim, é fundamental que seja realizada uma avaliação e um escrutínio relativamente aos conteúdos disseminados na Internet, definindo-se critérios para a sua avaliação e promovendo um maior destaque a sítios eletrónicos de associações e instituições de saúde pública (19).

A pesquisa por informação na consulta com o médico foi a segunda fonte de informação mais frequente. Os colaboradores que referiram obter informação nesta fonte apresentaram maiores conhecimentos na secção relativa à relação dieta-doença. Na verdade, os profissionais de saúde têm conhecimentos sobre saúde e sobre a causa das doenças, para além da compreensão relativamente aos comportamentos alimentares e as suas implicações para a saúde, justificando, assim maiores conhecimentos sobre alimentação (18).

A consulta com o nutricionista ou dietista também surge como fonte de informação, mas só em quinto lugar com uma percentagem de cerca de $19 \%$. Os colaboradores que referiram obter informação através de um nutricionista apresentaram maiores conhecimentos sobre recomendações dietéticas. Estes resultados poderão dever-se ao facto de que, numa consulta de nutrição, há espaço para a educação alimentar e informação nutricional, já que é a base para uma melhoria do estado nutricional e de bem-estar geral do indivíduo. Na literatura científica, encontram-se resultados similares a estes (29).

Para além disso, é importante referir que nem todos os indivíduos que apresentam um nível de conhecimentos alimentares elevado praticam, obrigatoriamente, hábitos saudáveis, já que pode existir discrepância entre conhecimentos, intenções e comportamentos. Esta ideia vai ao encontro ao descrito na literatura: as intervenções que consideram fatores psicológicos, como a motivação para a mudança de hábitos e fatores ambientais irão promover, mais eficazmente, hábitos saudáveis (30-32). Os resultados do presente estudo evidenciam que o nível de conhecimentos sobre alimentação desta população ativa não é elevado ( mediana $=65,5 \%$ ), principalmente na relação dieta-doença, justificando a necessidade de implementar intervenções a nível da promoção da literacia alimentar/nutricional, valorizando a vertente da educação alimentar e intervindo ao nível do ambiente alimentar no local de trabalho, que deverá estar alinhado com as recomendações nacionais propostas em 2019 pelo Programa Nacional para a Promoção da Alimentação Saudável (4, 32). Tal como todos os estudos, também este apresenta limitações. Tratando-se de um estudo exploratório, optou-se por proceder à recolha da informação do peso e da estatura por autorreportação dos inquiridos. Apesar de poder comprometer a precisão dos dados, considera-se que terá tido pouca influência nos resultados, devido à correlação muito forte entre os IMC calculados com base em valores de peso e altura autorreportados e medidos $(33,34)$. Sendo assim, considerou-se que, para os objetivos pretendidos, as vantagens suplantam os inconvenientes. O autorreporte do peso e da estatura e posterior cálculo do IMC também podem resultar numa imagem distorcida da realidade, uma vez que, relativamente ao peso, as mulheres têm tendência a sobrevalorizar valores baixos e a subvalorizar valores elevados enquanto os homens valorizam um peso corporal acima do real. A altura é habitualmente sobrestimada (35-38).

Outras limitações podem ser atribuídas à não obrigatoriedade de preenchimento do questionário, o que poderia levar à desistência dos inquiridos, uma vez que assumiriam esta atividade como mais uma tarefa, provavelmente não compreendendo as vantagens que este estudo poderia reverter para os próprios. Ainda, potenciais participantes mais saudáveis podem estar mais sensibilizados para colaborar, resultando num viés de participação. Apesar destas limitações, este estudo tem como pontos fortes o facto de poucos trabalhos sobre esta temática terem sido desenvolvidos em Portugal, contrastando com a relevância internacional deste tema, assim como o facto de contribuir para o diagnóstico da situação dos colaboradores de uma autarquia, servindo de ponto de partida para uma intervenção baseada na realidade constatada. 


\section{CONCLUSÕES}

Neste estudo concluiu-se que o nível de conhecimentos se revelou superior no sexo feminino, em indivíduos com menos idade, nos que possuem mais habilitações literárias e nos que se percecionam como mais saudáveis. Além disso, um maior nível de conhecimentos está, também, associado a um IMC mais baixo. Foi possível observar que existe um menor nível de conhecimentos no que concerne à relação dieta-doença. No que diz respeito à pesquisa por informação sobre alimentação/nutrição, esta é feita principalmente na Internet e/ou através de um médico. Apesar de a Internet ser a fonte preferencial de informação, os participantes que a utilizam não diferiam dos restantes em termos de conhecimentos. Por outro lado, os que referiram obter informação através de um nutricionista apresentaram níveis superiores de conhecimento.

\section{REFERÊNCIAS BIBLIOGRÁFICAS}

1. World Health Organization. World health statistics 2018: monitoring health for the SDGs, sustainable development goals. Geneva: World Health Organization; 2018. Contract No.: 2 Jul.

2. Ministério da Saúde. Retrato da Saúde. Portugal; 2018.

3. OECD, European Observatory on Health Systems and Policies. Portugal: Perfil de Saúde do País 2017, State of Health in the EU. Paris/European Observatory on Health Systems and Policies, Brussels: OECD; 2017.

4. Ministério da Saúde, Direção-Geral da Saúde. Programa para a Promoção da Alimentação Saudável. Lisboa: Direção-Geral da Saúde; 2019.

5. Institute for Health Metrics and Evaluation (IHME). Findings from the Global Burden of Disease Study 2017. Seattle, WA: IHME; 2018.

6. Programa Nacional para a Promoção da Alimentação Saudável. Alimentação Saudável: Desafios e Estratégias. 2018.

7. Afshin A, Penalvo J, Del Gobbo L, Kashaf M, Micha R, Morrish K, et al. CVD prevention through policy: a review of mass media, food/menu labeling, taxation/ subsidies, built environment, school procurement, worksite wellness, and marketing standards to improve diet. Current cardiology reports. 2015;17(11):98.

8. Wolf AM, Colditz GA. Current estimates of the economic cost of obesity in the United States. Obesity research. 1998;6(2):97-106.

9. Wanjek C. Food at Work: Workplace solutions for malnutrition, obesity and chronic diseases: International Labour Organization; 2005.

10. Fundação Francisco Manuel dos Santos. Pordata [website]. [citado em 201917 Jul]. Disponível em: https://www.pordata.pt/.

11. World Health Organization. Preventing noncommunicable diseases in the workplace through diet and physical activity: WHO/World Economic Forum report of a joint event. 2008.

12. Stokols D, Pelletier KR, Fielding JE. The ecology of work and health: research and policy directions for the promotion of employee health. Health education quarterly. 1996;23(2):137-58.

13. Allan J, Querstret D, Banas K, de Bruin M. Environmental interventions for altering eating behaviours of employees in the workplace: a systematic review. Obesity reviews : an official journal of the International Association for the Study of Obesity. 2017;18(2):214-26.

14. Souza J. Conhecimentos nutricionais: reprodução e validação do questionário: Universidade do Porto. Faculdade de Medicina. Instituto de Ciências Biomédicas Abel Salazar 2009.

15. Bonaccio M, Di Castelnuovo A, Costanzo S, De Lucia F, Olivieri M, Donati MB, et al. Nutrition knowledge is associated with higher adherence to Mediterranean diet and lower prevalence of obesity. Results from the Moli-sani study. Appetite. 2013;68:139-46. 16. Valmorbida JL, Goulart MR, Busnello FM, Pellanda LC. Nutritional knowledge and body mass index: A cross-sectional study. Revista da Associacao Medica Brasileira (1992). 2017;63(9):736-40.

17. Cash T, Desbrow B, Leveritt M, Ball L. Utilization and preference of nutrition information sources in Australia. Health Expectations. 2015;18(6):2288-95.
18. Quaidoo EY, Ohemeng A, Amankwah-Poku M. Sources of nutrition information and level of nutrition knowledge among young adults in the Accra metropolis. BMC public health. 2018;18(1):1323.

19. Fassier P, Chhim A-S, Andreeva VA, Hercberg S, Latino-Martel P, Pouchieu C, et al. Seeking health-and nutrition-related information on the Internet in a large population of French adults: results of the NutriNet-Santé study. British Journal of Nutrition. 2016;115(11):2039-46.

20. Wantland DJ, Portillo CJ, Holzemer WL, Slaughter R, McGhee EM. The effectiveness of Web-based vs. non-Web-based interventions: a meta-analysis of behavioral change outcomes. Journal of medical Internet research. 2004;6(4):e40.

21. Nabarette H, Romaneix F, Boyer C, Darmoni SJ, Remy P-L, Caniard E. Certification of health-related websites in France. Presse medicale (Paris, France: 1983). 2009;38(10):1476-83.

22. Scullard P, Peacock C, Davies P. Googling children's health: reliability of medical advice on the internet. Archives of disease in childhood. 2010;95(8):580-2.

23. Sutherland LA, Wildemuth B, Campbell MK, Haines PS. Unraveling the web: an evaluation of the content quality, usability, and readability of nutrition web sites. Journal of nutrition education and behavior. 2005;37(6):300-5.

24. Alfaro-Cruz L, Kaul I, Zhang Y, Shulman RJ, Chumpitazi BP. Assessment of Quality and Readability of Internet Dietary Information on Irritable Bowel Syndrome. Clinical Gastroenterology and Hepatology. 2019;17(3):566-7.

25. Fernandes A, Menezes J, Sá Sd, Vidal V, Silva Vd, Ferro-Lebres V. Recomendações dietéticas na diabetes: rigor da informação e qualidade dos sites portugueses. Revista Egitânia Sciencia. 2014(14):128-44.

26. Percheski C, Hargittai E. Health information-seeking in the digital age. Journal of American College Health. 2011;59(5):379-86.

27. Santana S, Pereira AS. Da utilização da internet para questões de saúde e doença em Portugal: possíveis repercussões na relação médico-doente. Acta Med Port. 2007;20(1):47-57.

28. Melo AR. Determinantes dos conhecimentos nutricionais: fontes de informação e crenças de saúde: Instituto Politécnico de Lisboa, Escola Superior de Tecnologia da Saúde de ...; 2016.

29. Spronk I, Heaney SE, Prvan T, O'Connor HT. Relationship between general nutrition knowledge and dietary quality in elite athletes. International journal of sport nutrition and exercise metabolism. 2015;25(3):243-51.

30. Geaney F, Fitzgerald S, Harrington JM, Kelly C, Greiner BA, Perry IJ. Nutrition knowledge, diet quality and hypertension in a working population. Preventive medicine reports. 2015;2:105-13.

31. Adriaanse MA, Vinkers CD, De Ridder DT, Hox JJ, De Wit JB. Do implementation intentions help to eat a healthy diet? A systematic review and meta-analysis of the empirical evidence. Appetite. 2011;56(1):183-93.

32. Geaney F, Kelly C, Greiner BA, Harrington JM, Perry IJ, Beirne P. The effectiveness of workplace dietary modification interventions: a systematic review. Preventive medicine. 2013;57(5):438-47.

33. Fonseca MJM, Faerstein E, Chor D, Lopes CS. Validity of self-reported weight and height and the body mass index within the" Pro-saude" study. Revista de saude publica. 2004;38:392-8.

34. Chor D, Coutinho ESF, Laurenti R. Reliability of self-reported weight and height among state bank employees. Revista de Saúde Pública. 1999;33(1):16-23.

35. Hill A, Roberts J. Body mass index: a comparison between self-reported and measured height and weight. Journal of Public Health. 1998;20(2):206-10.

36. Ramos E, Barros H. Self-reporting of weight and height. Effects in risk assessment. Rév Epidémiol Santé Publ. 1998;46(suppl 1):S26.

37. Ramos E, Lopes C, Oliveira A, Barros H. Unawareness of weight and height-the effect on self-reported prevalence of overweight in a population-based study. JNHAThe Journal of Nutrition, Health and Aging. 2009;13(4):310-4.

38. Jeffery RW. Bias in reported body weight as a function of education, occupation, health and weight concern. Addictive Behaviors. 1996;21(2):217-22. 